\title{
INERTIA SETS FOR GRAPHS ON SIX OR FEWER VERTICES*
}

\author{
WAYNE BARRETT ${ }^{\dagger}$, CAMILLE JEPSEN ${ }^{\ddagger}$, ROBERT LANG§ ${ }^{\S}$ EMILY MCHENRY $₫$, \\ CURTIS NELSON ${ }^{\ddagger}$, AND KAYLA OWENS"
}

\begin{abstract}
Let $G$ be an undirected graph on $n$ vertices and let $S(G)$ be the set of all real symmetric $n \times n$ matrices whose nonzero off-diagonal entries occur in exactly the positions corresponding to the edges of $G$. The inverse inertia problem for $G$ asks which inertias can be attained by a matrix in $S(G)$, a question which was previously answered when $G$ is a tree. In this paper, a number of new techniques are developed in order to be able to determine possible inertias of general graphs: covers with cliques, covers with cliques and clique-stars, and the graph operations of edge subdivision, edge deletion, joins, and unions. Because most of the associated theorems require additional hypotheses, definitive criteria that apply to all graphs cannot be provided. Nevertheless, these results are strong enough to be able to determine the inertia set of each graph on 6 or fewer vertices and can be applied to many graphs with larger order as well. One consequence of the 1-6 vertex results is the fact that all of these graphs have balanced inertia. It is also mentioned which of these results guarantee or preserve balanced inertia, and explain how to modify them to include Hermitian matrices.
\end{abstract}

Key words. Balanced inertia, Combinatorial matrix theory, Graph, Hermitian, Inertia, Inverse inertia problem, Minimum rank, Symmetric.

AMS subject classifications. 05C05, 05C50, 15A03, $15 \mathrm{~A} 57$.

1. Introduction. One area of combinatorial matrix theory asks the question: what can be known about symmetric matrices with specific zero patterns? Because every symmetric matrix with a particular zero pattern can be represented by a graph, graphs provide a convenient and concise description of such matrices. The symbiotic relationship between graphs and matrices aids particularly in the characterization of eigenvalues of matrices with prescribed zero patterns. Combinatorial matrix theory utilizes this interrelation as an approach to the widely studied inverse eigenvalue problem.

*Received by the editors July 31, 2009. Accepted for publication December 20, 2009. Handling Editor: Richard A. Brualdi. This research was conducted during the summer of 2009 as part of the Brigham Young University REU program and was supported by NSF grant DMS-0755422.

${ }^{\dagger}$ Department of Mathematics, Brigham Young University, Provo, Utah 84602, United States (wayne@math.byu.edu).

$\ddagger$ Undergraduates, Brigham Young University, Provo, Utah, 84602 (jepsencamille@gmail.com, curtisgn@gmail.com).

§Undergraduate, Florida Atlantic University, 777 Glades Rd, Boca Raton, FL 33431 (rlang6@fau.edu).

ฯ Undergraduate, Xavier University, 3800 Victory Parkway, Cincinnati, Ohio 45207

(mchenrye@xavier.edu).

" Masters Degree, Brigham Young University (kayla.d.owens@gmail.com). 
If $G$ is a graph, then we call $S(G)$ the set of all real symmetric $n \times n$ matrices $A=\left[a_{i j}\right]$ for which $a_{i j} \neq 0, i \neq j$, if and only if $i j$ is an edge of $G$. No restrictions are placed on the diagonal entries. The inverse eigenvalue problem asks: given a graph $G$ on $n$ vertices and numbers $\lambda_{1}, \lambda_{2}, \ldots, \lambda_{n}$, is there a matrix $A \in S(G)$ such that the eigenvalues of $A$ are exactly these numbers? Each year many papers are published on a wide variety of inverse eigenvalue problems. However, our combinatorial instance of this problem is difficult to solve. A simplification of the inverse eigenvalue problem is the inverse inertia problem.

The inverse inertia problem asks: given a graph $G$ on $n$ vertices and an ordered triple $(r, s, t)$ of nonnegative integers with $r+s+t=n$, is there a matrix $A \in S(G)$ such that $A$ has $r$ positive eigenvalues, $s$ negative eigenvalues and $t$ eigenvalues equal to 0 ? This problem allows for the characterization of possible eigenvalues of matrices in $S(G)$ without knowing their exact values. Given a matrix $A \in S(G)$ the ordered triple $(\pi(A), \nu(A), \delta(A))$ is called the inertia of $A$, where $\pi(A)$ is the number of positive eigenvalues of $A, \nu(A)$ is the number of negative eigenvalues of $A$, and $\delta(A)$ is the multiplicity of the eigenvalue 0 of $A$. The partial inertia of $A$, denoted $\operatorname{pin}(A)$, is the pair $(\pi(A), \nu(A))$. The inertia set of $G$ is the set of all partial inertias of matrices in $S(G)$ and is denoted $\mathcal{I}(G)$.

The inverse inertia problem is a refinement of the minimum rank problem. The minimum rank of a graph $G$, denoted $\operatorname{mr}(G)$, is the smallest rank over all matrices in $S(G)$. The minimum rank restricts possible inertias of matrices in $S(G)$ as follows. If $G$ is a graph on $n$ vertices and $A \in S(G)$, then $\pi(A)+\nu(A)=\operatorname{rank}(A)$. Because $\operatorname{rank}(A) \geq \operatorname{mr}(G)$, it follows that $\pi(A)+\nu(A) \geq \operatorname{mr}(G)$.

Barrett, Hall, and Loewy [4] determined the inertia sets for trees and graphs with cut vertices. In this paper we detail a sufficient number of new and constructive techniques we have developed to determine the inertia sets of all graphs on six or fewer vertices (see Table 9.1). The graph numbers correspond to those used in [10]. The various sections correspond to these techniques, and each graph whose inertia set is determined by a specific technique is also listed in that section. We consider the minimum ranks and minimum positive semidefinite ranks of these graphs to be well known and will seldom give reasons for these. The minimum ranks and minimum positive semidefinite ranks are catalogued at www.aimath.org/pastworkshops/matrixspectrum.html and in [6]. Although we only consider connected graphs, the inertias of disconnected graphs follow from Observation 4.1 in [4] which states that if $G$ is a graph with components $G_{1}, \ldots, G_{k}$, then an ordered pair is in $\mathcal{I}(G)$ if and only if it is a sum of ordered pairs belonging to $\mathcal{I}\left(G_{1}\right), \ldots, \mathcal{I}\left(G_{k}\right)$.

After completing our list we turn to the concept of balanced inertia introduced in [2]. We observe that all graphs on six or fewer vertices have balanced inertia and identify a few graph classes with balanced inertia. We next explain that for 
the Hermitian version of the inverse inertia problem one obtains the same results for graphs on six or fewer vertices as for the real symmetric case. We conclude with a few open problems.

\section{Definitions.}

\subsection{Matrix theory.}

Definition 2.1. Given an $n \times n$ real symmetric matrix $A$, the inertia of $A$ is the ordered triple $(\pi(A), \nu(A), \delta(A))$, where $\pi(A)$ is the number of positive eigenvalues of $A, \nu(A)$ is the number of negative eigenvalues of $A$, and $\delta(A)$ is the multiplicity of 0 as an eigenvalue of $A$.

Then $\pi(A)+\nu(A)+\delta(A)=n$ and $\pi(A)+\nu(A)=\operatorname{rank}(A)$.

If the order of $A$ is known, then we lose no information by discarding the third number of the triple.

Definition 2.2. Given a real symmetric matrix $A$, the partial inertia of $A$ is the ordered pair $(\pi(A), \nu(A))$, written $\operatorname{pin}(A)$.

Definition 2.3. Given a graph $G$ on $n$ vertices, let $S(G)$ be the set of all real symmetric $n \times n$ matrices $A=\left[a_{i j}\right]$ such that $a_{i j} \neq 0, i \neq j$, if and only if $i j$ is an edge of $G$. Let $S_{+}(G)$ be the subset of $S(G)$ consisting of all positive semidefinite matrices in $S(G)$. Then the minimum rank of $G$ is

$$
\operatorname{mr}(G)=\min _{A \in S(G)}\{\operatorname{rank}(A)\}
$$

The minimum positive semidefinite rank of $G$ is

$$
\operatorname{mr}_{+}(G)=\min _{A \in S_{+}(G)}\{\operatorname{rank}(A)\}
$$

The maximum nullity of $G$ is

$$
M(G)=\max _{A \in S(G)}\{\operatorname{nullity}(A)\} .
$$

The maximum positive semidefinite nullity of $G$ is

$$
M_{+}(G)=\max _{A \in S_{+}(G)}\{\operatorname{nullity}(A)\} .
$$

Note that $\operatorname{mr}(G)+M(G)=n$ and $\mathrm{mr}_{+}(G)+M_{+}(G)=n$.

Definition 2.4. Given a graph $G$, the inertia set $\mathcal{I}(G)$ is the set of all possible partial inertias of matrices in $S(G)$. That is,

$$
\mathcal{I}(G)=\{(r, s) \mid \operatorname{pin}(A)=(r, s) \text { for some } A \in S(G)\} .
$$


Note that if $(r, s) \in \mathcal{I}(G)$, then $\operatorname{mr}(G) \leq r+s \leq n$. Also $\mathrm{mr}_{+}(G)$ is the smallest integer $r$ such that $(r, 0)$ is in $\mathcal{I}(G)$.

Definition 2.5. The minimum rank line of a graph $G$ consists of all points $(r, s) \in \mathcal{I}(G)$ such that $r+s=\operatorname{mr}(G)$.

ObSERVATion 2.6. The minimum rank line is nonempty.

Definition 2.7. Let $\mathbb{N}$ be the set of nonnegative integers, and let $\mathbb{N}^{2}=\mathbb{N} \times \mathbb{N}$. The $k$-line is defined to be the set $\left\{(r, s) \in \mathbb{N}^{2} \mid r+s=k\right\}$. We define the set $\mathbb{N}_{\leq k}^{2}=\left\{(r, s) \in \mathbb{N}^{2}: r+s \leq k\right\}$.

It is known from Proposition 2.3 in [4] that for a graph $G$ on $n$ vertices, $\mathcal{I}(G)$ contains the $(n-1)$-line and $n$-line.

Definition 2.8. Let $m$ and $n$ be non-negative integers with $m \leq n$. When plotted as points in $\mathbb{R}^{2}$, the set

$$
\left\{(r, s) \in \mathbb{N}^{2} \mid m \leq r+s \leq n\right\}
$$

forms a trapezoid. We denote this set by $\mathrm{T}[m, n]$.

ObSERVATion 2.9. For any graph $G$ on $n$ vertices $\mathcal{I}(G) \subseteq \mathrm{T}[\operatorname{mr}(G), n]$.

Definition 2.10. Let $G$ be a graph on $n$ vertices. If $\mathcal{I}(G)=\mathrm{T}[k, n]$ for some nonnegative integer $k$, we say that $\mathcal{I}(G)$ is a trapezoid.

ObSERVATION 2.11. If $\mathcal{I}(G)$ is a trapezoid then $\mathcal{I}(G)=\mathrm{T}[\operatorname{mr}(G), n]$.

Definition 2.12. Let $G$ be a graph. Then $\mathcal{I}(G) \rightarrow$ is the set that results from adding $(1,0)$ to each element of $\mathcal{I}(G)$. Similarly, $\mathcal{I}(G)^{\uparrow}$ is the set that results from adding $(0,1)$ to each element of $\mathcal{I}(G)$.

Definition 2.13. If $Q$ is a subset of $\mathbb{N}^{2}$ and $n$ is a positive integer, we let

$$
[Q]_{n}=Q \cap \mathbb{N}_{\leq n}^{2}
$$

Definition 2.14. If $Q$ is a subset of $\mathbb{N}^{2}$, we define the northeast expansion of $Q$ as

$$
Q^{\nearrow}=Q+\mathbb{N}^{2}
$$




\subsection{Graph theory.}

Definition 2.15. A set of vertices in a graph $G$ is an independent set if its vertices are pairwise non-adjacent. The independence number of $G$, denoted $\alpha(G)$, is the size of the largest independent set in $G$.

Definition 2.16. A clique in a graph is a set of vertices which are pairwise adjacent. A complete graph is a graph whose vertex set forms a clique. The complete graph on $n$ vertices is notated $K_{n}$.

Definition 2.17. Given two graphs $G$ and $H$, the union of $G$ and $H$ is the graph $(V(G) \cup V(H), E(G) \cup E(H))$ and is written $G \cup H$. Note that the vertex sets of $G$ and $H$ need not be disjoint.

Definition 2.18. Given two graphs $G$ and $H$ with $V(G) \cap V(H)=\emptyset$, the join of $G$ and $H$, written $G \vee H$, is the graph with vertex set $V(G) \cup V(H)$ and edge set

$$
E(G) \cup E(H) \cup\{u v \mid u \in V(G) \text { and } v \in V(H)\} .
$$

Definition 2.19. We abbreviate the disjoint union $K_{1} \cup \ldots \cup K_{1}$ ( $n$ times) to $n K_{1}$. So $n K_{1}=K_{n}^{c}$, the graph consisting of $n$ isolated vertices.

DeFinition 2.20. A bipartite graph is a graph whose vertices can be partitioned into two independent sets. Given $m, n \in \mathbb{N}$, the graph $m K_{1} \vee n K_{1}$ is called a complete bipartite graph and is written $K_{m, n}$. The graph $K_{m} \vee n K_{1}, m \geq 1$ and $n \geq 2$, is called a clique-star, and is written $K S_{m, n}$.

Definition 2.21. A graph $G$ is chordal if there are no induced cycles, $C_{k}$, where $k \geq 4$.

Definition 2.22. Let $G$ be a graph. Then a cover of $G$ is a set of subgraphs of $G$ such that the union of the edge sets is equal to $E(G)$.

Definition 2.23. A clique cover of $G$ is a cover of $G$ consisting of only cliques. The clique cover number of $G$, written $\operatorname{cc}(G)$, is the smallest number of cliques in a clique cover of $\mathrm{G}$.

Definition 2.24. Let $G$ be a graph. Then a clique/clique-star cover of $G$ is a cover of $G$ consisting of only cliques and clique-stars.

DEFINITION 2.25. If $G$ is a graph and $v w \in E(G)$, subdividing $v w$ is the action of creating a new graph $G_{e}$ from $G$ by adding a new vertex $u$, and adjusting the edge set as shown:

$$
G_{e}=(V(G) \cup\{u\},(E(G) \backslash\{v w\}) \cup\{u v, u w\}) .
$$


3. Previous results and lemmata. The following two results are Corollary 2.7 and Theorem 3.6 from [6].

Theorem 3.1. For a connected graph $G, \mathrm{mr}_{+}(G) \geq \alpha(G)$.

TheOREm 3.2. Let $G$ be a connected chordal graph on $n \geq 2$ vertices. Then $\mathrm{mr}_{+}(G)=\operatorname{cc}(G)$.

The following is Lemma 2.11 from [7] (see the first definition in [7] for the significance of extra edges).

Lemma 3.3. Let $G=(V, E)$ be a graph and $v$ be a vertex with exactly two neighbors $r_{1}$ and $r_{2}$. If $v$ is connected to both neighbors by single edges, then

$$
M_{+}(G)=M_{+}(H)
$$

where $H$ is the graph obtained from $G-v$ by connecting $r_{1}$ and $r_{2}$ by an additional edge.

Assuming $H$ has $n$ vertices, this is equivalent to $n+1-\mathrm{mr}_{+}(G)=n-\mathrm{mr}_{+}(H)$ or $\mathrm{mr}_{+}(G)=\mathrm{mr}_{+}(H)+1$.

Note that if $r_{1}$ and $r_{2}$ are not adjacent in $G$, then $e=r_{1} r_{2}$ is an edge in $H$ and $G=H_{e}$. Consequently we have the following.

COROLlary 3.4. If $G$ is a graph with edge e then

$$
\mathrm{mr}_{+}\left(G_{e}\right)=\mathrm{mr}_{+}(G)+1
$$

The following lemma is a weaker result for the minimum rank of $G_{e}$ and is labeled as Lemma 2.1 in both [8] and [3].

LEMMA 3.5. If $G$ is a graph with edge e, then

$$
\operatorname{mr}(G) \leq \operatorname{mr}\left(G_{e}\right) \leq \operatorname{mr}(G)+1 .
$$

We will also need Observation 1 and Theorem 11 from [5].

Observation 3.6. A connected graph $G$ on 2 or more vertices has $\operatorname{mr}(G)=1$ if and only if it is complete.

TheOREM 3.7. Given a connected graph $G$, the following are equivalent.

1. $\mathrm{mr}_{+}(G) \leq 2$.

2. $G^{c}$ is the union of complete bipartite graphs.

3. $G$ is $\left(K_{3}^{c}, P_{4}\right)$-free. $\left[G\right.$ is $P_{4}$-free with $\left.\alpha(G) \leq 2\right]$.

The following 4 results are Observation 2.7, Proposition 1.4, Lemma 1.1 and Theorem 7.1 from [4]. 
Observation 3.8. Let $G$ be a graph. If $(r, s) \in \mathcal{I}(G)$, then $(s, r) \in \mathcal{I}(G)$ also.

Proposition 3.9. Let $A, B$, and $C$ be real symmetric $n \times n$ matrices with $A+B=$ $C$ and let $A(i)$ be a principal submatrix of $A$ of size $(n-1) \times(n-1)$ obtained by deleting the $i^{\text {th }}$ row and column. Then we have the following properties:

Interlacing $\pi(A)-1 \leq \pi(A(i)) \leq \pi(A)$ and $\nu(A)-1 \leq \nu(A(i)) \leq \nu(A)$.

Subadditivity $\pi(C) \leq \pi(A)+\pi(B)$ and $\nu(C) \leq \nu(A)+\nu(B)$.

Lemma 3.10 (Northeast Lemma). Let $G$ be a graph on $n$ vertices and suppose that $A \in S(G)$ with $\operatorname{pin}(A)=(\pi, \nu)$. Then for every pair of integers $r \geq \pi$ and $s \geq \nu$ satisfying $r+s \leq n$, there exists a matrix $B \in S(G)$ with $\operatorname{pin}(B)=(r, s)$.

TheOrem 3.11. Let $G$ be a graph and let $M \in S(G)$ be a real symmetric matrix with partial inertia $(k, 0), k>1$. Then there exists a matrix $M^{\prime} \in S(G)$ with partial inertia $(r, s)$ satisfying $r<k$ and $s<k$.

COROLlary 3.12. Let $G$ be a graph and suppose that $(2,0) \in \mathcal{I}(G)$. Then $(1,1) \in \mathcal{I}(G)$.

LEMma 3.13. For any positive integer $n$, there exists an orthogonal $n \times n$ matrix with all nonzero entries.

Proof. The case $n=1$ is trivial. If $n=2$, we can use the matrix

$$
\frac{1}{\sqrt{2}}\left[\begin{array}{cc}
1 & 1 \\
1 & -1
\end{array}\right]
$$

Thus we may assume $n \geq 3$.

The matrix $Q=I_{n}-\frac{2}{n} J_{n}$, where $J_{n}$ is the $n \times n$ all ones matrix, is symmetric, and

$$
\begin{aligned}
Q^{T} Q & =\left(I_{n}-\frac{2}{n} J_{n}\right)^{2} \\
& =I_{n}-\frac{4}{n} J_{n}+\frac{4}{n^{2}} J_{n}^{2} \\
& =I_{n}-\frac{4}{n} J_{n}+\frac{4}{n^{2}} n J_{n} \\
& =I_{n} .
\end{aligned}
$$

So $Q$ is orthogonal. Since $n \neq 2$, every entry of this matrix is nonzero.

\section{Basic graphs and associated covers.}

4.1. Complete graphs. For the case $G=K_{1}$, it is clear that $\mathcal{I}\left(K_{1}\right)=\mathrm{T}[0,1]$.

Proposition 4.1. If $G=K_{n}, n \geq 2$, then $\mathcal{I}(G)=\mathrm{T}[1, n]$. 
Proof. Since $\operatorname{mr}(G)=1$ by Observation 3.6, $\{(1,0),(0,1)\} \in \mathcal{I}(G)$ by Observations 2.6 and 3.8. By the Northeast Lemma, $\mathcal{I}(G)=\mathrm{T}[1, n]$.

\subsection{Complete bipartite graphs.}

THEOREM 4.2. If $m, n \in \mathbb{N}$, with $m \leq n$ then

$$
\mathcal{I}\left(K_{m, n}\right)=\mathrm{T}[n, m+n] \cup\left[\{(1,1)\}^{\nearrow}\right]_{m+n}
$$

Proof. By the Northeast Lemma, it suffices to show that $(1,1) \in \mathcal{I}\left(K_{m, n}\right)$ and $\mathrm{mr}_{+}\left(K_{m, n}\right)=n$. Let $X$ and $Y$ be the bipartite sets of $K_{m, n}$ with cardinalities $m$ and $n$ respectively. Assume the vertices of $Y$ precede all vertices of $X$. Let $J_{n, m}$ be the $n \times m$ all ones matrix and consider the matrix

$$
\left[\begin{array}{cc}
0 & J_{n, m} \\
J_{m, n} & 0
\end{array}\right] \in S\left(K_{m, n}\right) .
$$

Note that the rank of this matrix is 2 , and since the trace is 0 , it must have one positive and one negative eigenvalue. So, $(1,1) \in \mathcal{I}\left(K_{m, n}\right)$.

By Theorem 3.1, $\mathrm{mr}_{+}\left(K_{m, n}\right) \geq \alpha\left(K_{m, n}\right)$. Since $Y$ is an independent set of cardinality $n$, we have $\mathrm{mr}_{+}\left(K_{m, n}\right) \geq n$. We now construct the required matrix. By Lemma 3.13, let $Q$ be an $n \times n$ orthogonal matrix with all non-zero entries. Let $B$ be the matrix obtained from taking the first $m$ columns of $Q$. Then consider the matrix

$$
\left[\begin{array}{c}
I_{n} \\
B^{T}
\end{array}\right]\left[\begin{array}{ll}
I_{n} & B
\end{array}\right]=\left[\begin{array}{cc}
I_{n} & B \\
B^{T} & I_{m}
\end{array}\right] \in S\left(K_{m, n}\right) .
$$

This matrix is positive semidefinite and its rank is $n$. So $\operatorname{mr}_{+}\left(K_{m, n}\right) \leq n$. Therefore, $\mathrm{mr}_{+}\left(K_{m, n}\right)=n$.

\subsection{Clique-stars.}

LEMMA 4.3. Every clique-star $K S_{m, n}$ has minimum rank equal to 2 and $(1,1) \in$ $\mathcal{I}\left(K S_{m, n}\right)$.

Proof. Note that every clique-star $K S_{m, n}$ can be written as a complete graph on $m+n$ vertices minus the edge set of a complete graph on $n$ vertices. It follows that,

$$
A=\left[\begin{array}{cc}
J_{m} & J_{m, n} \\
J_{n, m} & 0_{n}
\end{array}\right] \in S\left(K S_{m, n}\right) .
$$

Since $K S_{m, n}$ is not a complete graph, $2 \leq \operatorname{mr}\left(K S_{m, n}\right) \leq \operatorname{rank}(A)=2$. Also $\operatorname{pin}(A)=(1,1) \in \mathcal{I}(G) . \square$ 
Theorem 4.4. If $G=K S_{m, n}$ then $\mathcal{I}(G)=\mathrm{T}[n, n+m] \cup\left[\{(1,1)\}^{\nearrow}\right]_{n+m}$.

Proof. Since $G$ is a clique-star, it is a chordal graph. So, by Theorem 3.2, $\mathrm{mr}_{+}(G)=\operatorname{cc}(G)=n$. By Lemma 4.3 and the Northeast Lemma, the point $(1,1)$ and all points northeast are included. Therefore, $\mathcal{I}(G)=\mathrm{T}[n, n+m] \cup\left[\{(1,1)\}^{\nearrow}\right]_{n+m}$. $\mathrm{u}$

EXAmple 4.5. Consider $K S_{2,3}$ (G46).

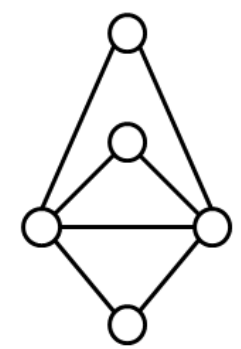

Then $\mathcal{I}\left(K S_{2,3}\right)=\mathrm{T}[3,5] \cup\left[\{(1,1)\}^{\nearrow}\right]_{5}$ and its inertia plot is the following figure.

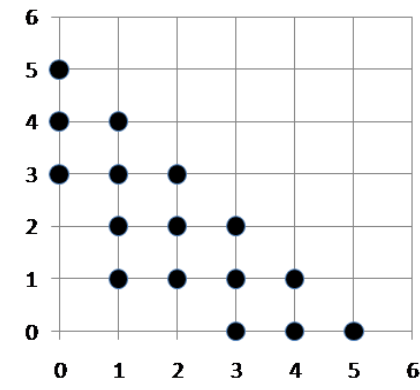

Each of the following graphs fall into one of the preceding classes so their inertia sets are determined: G1, G3, G7, G13, G18, G29, G44, G46, G52, G77, G146, G161, G175, G201, G208. Note that there are additional graphs that can be categorized as clique-stars, but for convenience they will be included in a following section.

4.4. Clique covers. Let $K$ be a clique in a graph on $n$ vertices. Define the $n \times n$ matrix $J_{K}$ by

$$
\left(J_{K}\right)_{i j}=\left\{\begin{array}{ll}
1 & \text { if } i, j \in K \\
0 & \text { otherwise }
\end{array} .\right.
$$

TheOREM 4.6. Let $G$ be a graph on $n$ vertices. If $\operatorname{mr}(G)=\operatorname{cc}(G)$, then $\mathcal{I}(G)=$ $\mathrm{T}[\operatorname{mr}(G), n]$.

Proof. Let $k=\operatorname{cc}(G)=\operatorname{mr}(G)$. Since $\mathcal{I}(G) \subseteq \mathrm{T}[k, n]$ by Observation 2.9, it suffices to show $\mathcal{I}(G) \supseteq \mathrm{T}[k, n]$. Let $M_{1}, M_{2}, \ldots, M_{k}$ be the cliques in a minimum 
clique cover of $G$ and let $(m, k-m)$ be on the minimum rank line of $G$ where $m \in \mathbb{N}$ such that $0 \leq m \leq k$.

Let $A_{i}=J_{M_{i}}$ for $i=1, \ldots, m$ and let $A_{i}=-(m+1) J_{M_{i}}$ for $i=m+1, \ldots, k$. ( $k=0$ and $k=m$ are allowed.) Let $A=A_{1}+A_{2}+\ldots+A_{k}$. Since an off-diagonal entry in $A_{1}+A_{2}+\ldots+A_{m}$ is at most $m$ and each non-zero off-diagonal entry in $A_{m+1}+\ldots+A_{k}$ is at most $-(m+1)$, every off-diagonal entry in $A$ corresponding to an edge in $G$ is non-zero. Therefore, $A \in S(G)$. By Subadditivity in Proposition 3.9, $\pi(A) \leq m$ and $\nu(A) \leq k-m$. Because $k=\operatorname{mr}(G) \leq \operatorname{rank}(A)=\pi(A)+\nu(A) \leq k$, $\pi(A)=m$ and $\nu(A)=k-m$. Therefore, $(m, k-m) \in \mathcal{I}(G)$.

By the Northeast Lemma $\mathcal{I}(G) \supseteq \mathrm{T}[k, n]$. $\square$

EXAMPle 4.7. Consider G40.

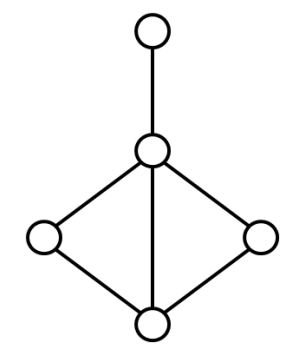

Because $\operatorname{mr}(G 40)=\operatorname{cc}(G 40)=3$, we know $\mathcal{I}(G 40)=\mathrm{T}[3,5]$.

Similarly, all of the inertia sets of the following graphs are determined by this theorem: G6, G14, G15, G17, G34, G35, G36, G40, G41, G42, G45, G47, G49, G51, G93, G94, G95, G97, G102, G111, G112, G113, G115, G117, G119, G120, G123, G130, G133, G134, G136, G137, G139, G142, G144, G148, G150, G152, G156, G157, G160, G163, G164, G165, G167, G177, G178, G179, G180, G181, G183, G191, G192, G193, G195, G200, G202, G205, G207. All of the inertia sets for these graphs are trapezoids.

\subsection{Clique/Clique-star covers.}

TheOrem 4.8. Let $G$ be a graph on $n$ vertices. Assume that $G$ has a cover with $p$ cliques and $q$ clique-stars with $p+2 q \leq n$. Then $(p-k+q, k+q) \in \mathcal{I}(G)$ for $k=0,1,2, \ldots, p$.

Proof. Let $N=K S_{m, n_{0}}=K_{m} \vee n_{0} K_{1}$ be a clique-star in $G$. Define the $n \times n$ matrix $H_{N}$ by

$$
\left(H_{N}\right)_{i j}=\left\{\begin{array}{ll}
1 & \text { if } i j \text { is an edge in } N \text { or } i=j \in V\left(K_{m}\right) \\
0 & \text { otherwise }
\end{array} .\right.
$$


Note that the rank of $H_{N}$ is 2 , as in Lemma 4.3, and the partial inertia is $(1,1)$. Let $M_{1}, M_{2}, \ldots, M_{p}$ be the cliques in the cover of $G$. Let $N_{1}, N_{2}, \ldots, N_{q}$ be the clique-stars in the cover of $G$.

Let $A_{i}=-J_{M_{i}}$ for $i=1, \ldots, k$ and $A_{i}=(k+1) J_{M_{i}}$ for $i=k+1, \ldots, p . \quad(k=0$ and $k=p$ are allowed.) Let $B_{j}=(k+1) H_{N_{j}}$ for $j=1, \ldots, q$. Let $A=A_{1}+A_{2}+$ $\ldots+A_{p}+B_{1}+B_{2}+\ldots+B_{q}$. Our construction ensures that if the $i, j$ entry is nonzero in any of $A_{1}, A_{2}, \ldots, A_{p}, B_{1}, B_{2}, \ldots, B_{q}$, it is nonzero in $A$. Therefore, $A \in S(G)$. By Subadditivity in Proposition 3.9, $\pi(A) \leq p-k+q$ and $\nu(A) \leq k+q$.

Since $p-k+q+k+q \leq n$, by the Northeast Lemma, $(p-k+q, k+q) \in \mathcal{I}(G)$.

Example 4.9. Consider G80.

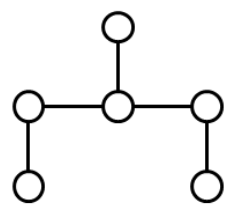

There is a cover of G80 with 2 cliques and one clique-star. Therefore, by Theorem 4.8, $(3,1),(2,2),(1,3) \in \mathcal{I}(G 80)$. Further, $\operatorname{mr}(G 80)=4$ and since G80 is chordal, $\mathrm{mr}_{+}(G 80)=c c(G 80)=5$ by Theorem 3.2. Therefore, $\mathcal{I}(G 80)=\mathrm{T}[5,6] \cup$ $\{(3,1),(2,2),(1,3)\}$.

The previous theorem and method in the example may be applied to determine the inertia sets of the graphs G79, G80, G81, G92, G100, G114, G135, G138, and G162.

\section{Graphs whose minimal positive semidefinite rank is 2 .}

TheOREm 5.1. Let $G \neq K_{n}$ be a connected graph on $n \geq 3$ vertices for which $G^{c}$ is a disjoint union of complete bipartite graphs. Then

$$
\mathcal{I}(G)=\mathrm{T}[2, n]
$$

Proof. The graph $G$ is not complete so $2 \leq \operatorname{mr}(G)$. By Theorem 3.7, $\mathrm{mr}_{+}(G) \leq 2$. Since $\operatorname{mr}(G) \leq \mathrm{mr}_{+}(G)$ we have $\operatorname{mr}(G)=\mathrm{mr}_{+}(G)=2$. Therefore, $(2,0) \in \mathcal{I}(G)$. By Corollary $3.12(1,1) \in \mathcal{I}(G)$. Therefore, $\mathrm{T}[2, n] \subseteq \mathcal{I}(G)$. By Observation 2.9, $\mathcal{I}(G) \subseteq \mathrm{T}[\operatorname{mr}(G), n]=\mathrm{T}[2, n]$.

This theorem may be applied to determine the inertia sets of graphs G50, G190, G199, G203, G204, and G206. None of these graphs is complete and their complements are $2 K_{2} \cup K_{1}, K_{2,2} \cup K_{2}, 2 P_{3}, K_{1} \cup K_{2} \cup P_{3}, 3 K_{2}$, and $2 K_{2} \cup 2 K_{1}$ respectively. 
Consequently, the inertia set of graph G50 $\left(W_{5}\right)$ is $\mathrm{T}[2,5]$ and the inertia set of each of graphs G190, G199, G203, G204, and G206 is T[2,6].

\section{Edge techniques.}

\subsection{Edge subdivision.}

Theorem 6.1 (Edge Subdivision Theorem for Partial Inertias). Let $G$ be a graph and $e$ be an edge of $G$. Let $G_{e}$ be the graph obtained from $G$ by subdividing the edge e. If $(r, s) \in \mathcal{I}(G)$, then $(r+1, s)$ and $(r, s+1)$ are contained in $\mathcal{I}\left(G_{e}\right)$.

Proof. Let $v, w$ be the vertices of $e$ and let $u$ be the new vertex in $G_{e}$ that is adjacent to $v$ and $w$. Let

$$
A=\left[\begin{array}{ccc}
d_{1} & a & b^{T} \\
a & d_{2} & c^{T} \\
b & c & B
\end{array}\right] \in S(G)
$$

with $\operatorname{pin}(A)=(r, s)$ and the first two rows and columns of $A$ labeled by $v$ and $w$ so that $a \neq 0$. If necessary perform a similarity by $\left[\begin{array}{cc}-1 & 0^{T} \\ 0 & I_{n-1}\end{array}\right]$ to ensure that $a$ is positive. Note that the inertia is a similarity invariant. Let

$$
A_{1}=\left[\begin{array}{cccc}
-a & -a & -a & 0 \\
-a & -a & -a & 0 \\
-a & -a & -a & 0 \\
0 & 0 & 0 & 0
\end{array}\right] .
$$

Note that $\operatorname{pin}\left(A_{1}\right)=(0,1)$. Let

$$
A_{e}=\left[\begin{array}{cccc}
0 & 0 & 0 & 0^{T} \\
0 & d_{1} & a & b^{T} \\
0 & a & d_{2} & c^{T} \\
0 & b & c & B
\end{array}\right]+\left[\begin{array}{cccc}
-a & -a & -a & 0 \\
-a & -a & -a & 0 \\
-a & -a & -a & 0 \\
0 & 0 & 0 & 0
\end{array}\right]=\left[\begin{array}{cccc}
-a & -a & -a & 0^{T} \\
-a & d_{1}-a & 0 & b^{T} \\
-a & 0 & d_{2}-a & c^{T} \\
0 & b & c & B
\end{array}\right]
$$

Then $A_{e} \in S\left(G_{e}\right)$. Subtracting row 1 from rows 2 and 3 yields

$$
\left[\begin{array}{cccc}
-a & -a & -a & 0^{T} \\
0 & d_{1} & a & b^{T} \\
0 & a & d_{2} & c^{T} \\
0 & b & c & B
\end{array}\right]
$$

and subtracting column 1 from columns 2 and 3 results in

$$
\left[\begin{array}{cccc}
-a & 0 & 0 & 0^{T} \\
0 & d_{1} & a & b^{T} \\
0 & a & d_{2} & c^{T} \\
0 & b & c & B
\end{array}\right]=[-a] \oplus A
$$


So, $\operatorname{pin}\left(A_{e}\right)=(r, s+1)$ and $(r, s+1) \in \mathcal{I}\left(G_{e}\right)$.

Since $(r, s) \in \mathcal{I}(G)$, we know $(s, r) \in \mathcal{I}(G)$ by Observation 3.8. Then by the proof above, $(s, r+1) \in \mathcal{I}\left(G_{e}\right)$, and by Observation 3.8 again, $(r+1, s) \in \mathcal{I}\left(G_{e}\right)$.

Corollary 6.2. Let $G$ be a graph such that $\mathcal{I}(G)$ is a trapezoid. If an edge e is subdivided and the minimum rank of the resulting graph $G_{e}$ increases, then $\mathcal{I}\left(G_{e}\right)$ is also a trapezoid.

Proof. Let $(r, s)$ be a point on the minimum rank line for $G_{e}$. Then either $r$ or $s$ is positive. This implies that either $(r, s-1)$ or $(r-1, s)$ is on the minimum rank line for $G$. Because $\mathcal{I}(G)$ is a trapezoid, $(r, s-1) \in \mathcal{I}(G)$ or $(r-1, s) \in \mathcal{I}(G)$. By the Edge Subdivision Theorem for Partial Inertias, $(r, s) \in \mathcal{I}\left(G_{e}\right)$.

COROLLARY 6.3. Let $G$ be a graph with edge e and let $G_{e}$ be the graph that results from the subdivision of $e$. Then $\mathcal{I}(G)^{\uparrow} \cup \mathcal{I}(G) \rightarrow \subseteq \mathcal{I}\left(G_{e}\right)$.

Proof. Let $(r, s) \in \mathcal{I}(G)^{\uparrow} \cup \mathcal{I}(G) \rightarrow$. Then $(r-1, s) \in \mathcal{I}(G)$ or $(r, s-1) \in \mathcal{I}(G)$. By the Edge Subdivision Theorem for Partial Inertias, if $(r-1, s) \in \mathcal{I}(G)$, then $(r, s) \in \mathcal{I}\left(G_{e}\right)$. Similarly if $(r, s-1) \in \mathcal{I}(G)$, then $(r, s) \in \mathcal{I}\left(G_{e}\right)$.

EXAmple 6.4. Consider G48.

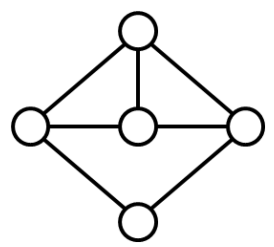

This is $K_{4}$ with one edge subdivision. By Lemma 3.5, $\operatorname{mr}\left(\left(K_{4}\right)_{e}\right)$ is either $\operatorname{mr}\left(K_{4}\right)$ or $\operatorname{mr}\left(K_{4}\right)+1$. Because G48 is not a complete graph, we know that $\operatorname{mr}(G 48)=$ $\operatorname{mr}\left(K_{4}\right)+1=2$. Therefore, by Corollary $6.2, \mathcal{I}(G 48)=T[2,5]$.

Graphs G151, G153, G154 are edge subdivisions of G48, each with minimum rank of 3. Therefore, the inertia sets of each of these graphs is $\mathrm{T}[3,6]$ by Corollary 6.2.

The inertia sets of the following graphs are determined by Corollary 6.2: G16, G31, G37, G38, G43, G48, G83, G96, G98, G99, G103, G104, G105, G118, G122, G124, G126, G127, G128, G140, G141, G147, G151, G153, G154, G166, G168, G169, G170, G171, G173, G185, G186, G194. Note that the inertia set of each of these graphs is a trapezoid.

Each of the graphs G30, G78, G129, G145 is an edge subdivision of a graph with lower minimum rank. Their inertia sets are determined by Corollary 6.3 and Corollary 3.4. None of the inertia sets of these graphs is a trapezoid. 
G121, G125, and G149 are edge subdivisions of G40, G41, and G47 respectively. Because $\operatorname{mr}(G 121)=3=\operatorname{mr}(G 40)$ we cannot use Corollary 6.2 to obtain $\mathcal{I}(G 121)$. However, by Corollary 3.4 we know $\mathrm{mr}_{+}(G 121)=\operatorname{mr}(G 40)+1=4$. By Observation 2.6 , the minimum rank line is nonempty so we must have $(1,2)$ or $(2,1)$ in $\mathcal{I}(G 121)$. However, by Observation 3.8 if one of these points is in $\mathcal{I}(G 121)$, both points are in $\mathcal{I}(G 121)$. Therefore, $\mathcal{I}(G 121)=\mathrm{T}[4,6] \cup\{(1,2),(2,1)\}$. By a similar argument, the inertia set of both G125 and G149 is also $\mathrm{T}[4,6] \cup\{(1,2),(2,1)\}$.

Corollary 6.2 can also be applied to determine the inertia sets of $P_{n}$ and $C_{n}$ for all $n$.

OBservation 6.5. For all positive integers $n, \mathcal{I}\left(P_{n}\right)=\mathrm{T}[n-1, n]$ and for all positive integers $n \geq 3, \mathcal{I}\left(C_{n}\right)=\mathrm{T}[n-2, n]$. The inertia set of $P_{n}$ was observed in [4] using a different method.

\subsection{Edge deletion.}

TheOREm 6.6 (Edge Deletion Theorem for Partial Inertias). Let $G$ be a graph and $e$ be an edge of $G$. Let $G \backslash e$ be the graph obtained from $G$ by removing the edge e. If $(r, s) \in \mathcal{I}(G)$, with $r+s \leq n-1$, then $(r+1, s)$ and $(r, s+1)$ are contained in $\mathcal{I}(G \backslash e)$.

Proof. Let $v, w$ be the vertices of $e$. Let

$$
A=\left[\begin{array}{ccc}
d_{1} & a & b^{T} \\
a & d_{2} & c^{T} \\
b & c & B
\end{array}\right] \in S(G)
$$

with $\operatorname{pin}(A)=(r, s)$ and the first two rows and columns of $A$ labeled by $v$ and $w$ so that $a \neq 0$. If necessary perform a similarity by $\left[\begin{array}{cc}-1 & 0^{T} \\ 0 & I_{n-1}\end{array}\right]$ to ensure that $a$ is positive. Let

$$
A_{1}=\left[\begin{array}{ccc}
-a & -a & 0 \\
-a & -a & 0 \\
0 & 0 & 0
\end{array}\right]
$$

Note that $\operatorname{pin}\left(A_{1}\right)=(0,1)$. Let

$$
A^{\prime}=A+A_{1}=\left[\begin{array}{ccc}
d_{1}-a & 0 & b^{T} \\
0 & d_{2}-a & c^{T} \\
b & c & B
\end{array}\right] \in S(G \backslash e) .
$$

Then $\operatorname{rank} A^{\prime} \leq \operatorname{rank} A+\operatorname{rank} A_{1}=\operatorname{rank} A+1$ 
Because $\operatorname{rank}(A)=r+s$, it follows that $\operatorname{rank}\left(A^{\prime}\right) \leq r+s+1$. By Proposition 3.9, $\pi\left(A^{\prime}\right) \leq \pi(A)+\pi\left(A_{1}\right)=r$ and $\nu\left(A^{\prime}\right) \leq \nu(A)+\nu\left(A_{1}\right)=s+1$. Since $r+s \leq n-1$, we have that $r+s+1 \leq n$, so by the Northeast Lemma, $(r, s+1) \in \mathcal{I}(G \backslash e)$.

Since $(r, s) \in \mathcal{I}(G)$, we know $(s, r) \in \mathcal{I}(G)$ by Observation 3.8. Then by the proof above, $(s, r+1) \in \mathcal{I}(G \backslash e)$, and by Observation 3.8 again, $(r+1, s) \in \mathcal{I}(G \backslash e)$.

Corollary 6.7. Let $G$ be a graph such that $\mathcal{I}(G)$ is a trapezoid. If an edge $e$ is deleted and the minimum rank of the resulting graph $G \backslash e$ increases, then $\mathcal{I}(G \backslash e)$ is also a trapezoid.

Proof. Proposition 2.1 of [9] states that $\operatorname{mr}(G \backslash e) \leq \operatorname{mr}(G)+1$. So we may say that $\operatorname{mr}(G \backslash e)=\operatorname{mr}(G)+1$. Let $(r, s)$ be a point on the minimum rank line for $G \backslash e$. Then either $r$ or $s$ is positive. This implies that either $(r, s-1)$ or $(r-1, s)$ is on the minimum rank line for $G$. Because $\mathcal{I}(G)$ is a trapezoid, $(r, s-1) \in \mathcal{I}(G)$ or $(r-1, s) \in \mathcal{I}(G)$. By the Edge Deletion Theorem for Partial Inertias, $(r, s) \in \mathcal{I}(G \backslash e)$.

COROLlary 6.8. Let $G$ be a graph with edge e and let $G \backslash e$ be the graph that results from the deletion of $e$. Then $\mathcal{I}(G)^{\uparrow} \cup \mathcal{I}(G) \rightarrow \subseteq \mathcal{I}(G \backslash e)$.

Proof. Let $(r, s) \in \mathcal{I}(G)^{\uparrow} \cup \mathcal{I}(G) \rightarrow$. Then $(r-1, s) \in \mathcal{I}(G)$ or $(r, s-1) \in \mathcal{I}(G)$. By the Edge Deletion Theorem for Partial Inertias if $(r-1, s) \in \mathcal{I}(G)$, then $(r, s) \in$ $\mathcal{I}(G \backslash e)$. Similarly if $(r, s-1) \in \mathcal{I}(G)$, then $(r, s) \in \mathcal{I}(G \backslash e)$.

Two graphs whose inertia sets are determined by the preceding argument are G188 and G189. Both graphs use G199 to determine their inertia sets.

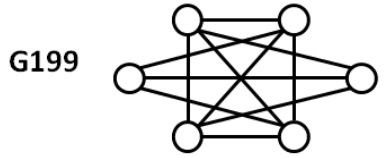

We use the fact from Section 5 that $\operatorname{mr}(G 199)=2$ and that $\mathcal{I}(G 199)=\mathrm{T}[2,6]$.

First, consider the graph G188. This graph is obtained by deleting the top horizontal edge of G199. An easy way to see this is the complement of G188 is a path on 6 vertices and deleting this edge would make the complement of the new graph a path on 6 vertices.

Since $\operatorname{mr}(G 188)=3$ and $\mathcal{I}(G 199)=\mathrm{T}[2,6]$, by Corollary 6.7 we have $\mathcal{I}(G 188)=$ $\mathrm{T}[3,6]$.

Now, consider the graph G189. This graph is obtained by deleting the rightmost vertical edge of G199. An easy way to see this is the complement of G189 is $K_{3} \cup K_{1,2}$ and deleting this edge would make the complement of the new graph $K_{3} \cup K_{1,2}$. 
It is known that $\operatorname{mr}(G 189)=2$ (see for example [5]). We have that the 2-line $\subseteq$ $\mathcal{I}(G 199)$ so by the Edge Deletion Theorem, the 3-line $\subseteq \mathcal{I}(G 189)$. However, this is not the entire inertia set for G189 as it was for G188. By Observation 2.6, we know there is at least one point on the minimum rank line. Because $\mathrm{mr}_{+}($G189) $\geq \alpha(G 189)=3$ and consequently the points $(2,0)$ and $(0,2) \notin \mathcal{I}(G 189)$, we must have $(1,1)$ in the inertia set. Thus, $\mathcal{I}(G 189)=\mathrm{T}[3,6] \cup\{(1,1)\}$.

\section{Joins.}

TheOREM 7.1. Let $G$ be a connected graph on $n \geq 2$ vertices. Then

$$
\left[\mathcal{I}\left(G \vee K_{1}\right)\right]_{n}=\mathcal{I}(G) .
$$

Proof. Forward Containment: Let $(r, s) \in\left[\mathcal{I}\left(G \vee K_{1}\right)\right]_{n}$, let $A \in S\left(G \vee K_{1}\right)$ with $\operatorname{pin}(A)=(r, s)$, and let $v$ be the vertex associated with $K_{1}$ in $G \vee K_{1}$. Then $A(v) \in S(G)$. So by Proposition $3.9 \pi(A(v)) \leq \pi(A)=r$ and $\nu(A(v)) \leq \nu(A)=s$. Since $(\pi(A(v)), \nu(A(v))) \in \mathcal{I}(G)$ and $r+s \leq n$, we have $(r, s) \in \mathcal{I}(G)$ by the Northeast Lemma.

Reverse Containment: Let $(r, s) \in \mathcal{I}(G)$ and let $A \in S(G)$ with $\operatorname{pin}(A)=(r, s)$. Since $A$ is connected, no row of $A$ is zero. Let $a_{1}, a_{2}, \ldots, a_{n}$ be the row vectors of $A$ and let $x=\left(x_{1}, x_{2}, \ldots, x_{n}\right)^{T}$ be any vector in $\mathbb{R}^{n}$. Then $a_{1} \cdot x=0, a_{2} \cdot x=0, \ldots, a_{n} \cdot x=0$ are the equations of $n$ hyperplanes through the origin in $\mathbb{R}^{n}$. Let $y$ be a vector that lies on none of these hyperplanes so $a_{i} \cdot y \neq 0$ for $i=1, \ldots, n$. Let $B=\left[\begin{array}{cc}A & A y \\ y^{T} A & y^{T} A y\end{array}\right]$. Then $B \in S\left(G \vee K_{1}\right)$. Since $A y$ is a linear combination of the columns of $A$ and $\left[\begin{array}{ll}y^{T} A & y^{T} A y\end{array}\right]$ is a linear combination of the rows of $\left[\begin{array}{ll}A & A y\end{array}\right]$, we have $\operatorname{rank}(A)=$ $\operatorname{rank}(B)$. By Proposition 3.9, $\pi(A) \leq \pi(B)$ and $\nu(A) \leq \nu(B)$. This implies $\operatorname{rank}(A)=$ $\pi(A)+\nu(A) \leq \pi(B)+\nu(B)=\operatorname{rank}(B)=\operatorname{rank}(A)$. Therefore, $\pi(B)=\pi(A)=r$ and $\nu(B)=\nu(A)=s$. Since $r+s \leq n,(r, s)=(\pi(B), \nu(B)) \in\left[\mathcal{I}\left(G \vee K_{1}\right)\right]_{n}$.

We thank Raphael Loewy for the essential idea in the proof of the reverse containment.

Corollary 7.2. Let $G$ be a connected graph on $n$ vertices. Then $\operatorname{mr}(G)=$ $\operatorname{mr}\left(G \vee K_{1}\right)$ and $\mathrm{mr}_{+}(G)=\mathrm{mr}_{+}\left(G \vee K_{1}\right)$.

Example 7.3. Consider $W_{6}(\mathrm{G} 187)$

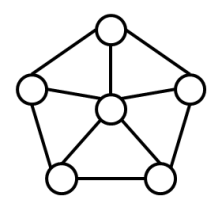


which is the join of $C_{5}$ (G38) and $K_{1}$. The inertia set of $C_{5}$ was determined in Section 6. Then by Theorem $7.1,\left[\mathcal{I}\left(W_{6}\right)\right]_{5}=\mathcal{I}\left(C_{5}\right)=\mathrm{T}[3,5]$. It follows that $\mathcal{I}\left(W_{6}\right)=\mathrm{T}[3,6]$.

The inertia sets of G187 and G197 are determined by Theorem 7.1. We could have also used Theorem 7.1 to determine the inertia sets of 28 other connected graphs on 6 or fewer vertices including all clique-stars that are not trees.

\section{Unions.}

TheOREm 8.1. Let $G$ and $H$ be connected graphs with $\mathcal{I}(G)=\mathrm{T}[\operatorname{mr}(G),|G|]$ and $\mathcal{I}(H)=\mathrm{T}[\operatorname{mr}(H),|H|]$. If $\operatorname{mr}(G \cup H)=\operatorname{mr}(G)+\operatorname{mr}(H)$, then

$$
\mathcal{I}(G \cup H)=\mathrm{T}[\operatorname{mr}(G)+\operatorname{mr}(H),|G \cup H|] .
$$

Proof. By Observation 2.9 and the hypothesis,

$$
\mathcal{I}(G \cup H) \subseteq \mathrm{T}[\operatorname{mr}(G \cup H),|G \cup H|]=\mathrm{T}[\operatorname{mr}(G)+\operatorname{mr}(H),|G \cup H|] .
$$

To prove $\mathcal{I}(G \cup H) \supseteq \mathrm{T}[\operatorname{mr}(G)+\operatorname{mr}(H),|G \cup H|]$, let $(r, s) \in \mathrm{T}[\operatorname{mr}(G)+\operatorname{mr}(H),|G \cup H|]$ such that $r+s=\operatorname{mr}(G)+\operatorname{mr}(H)$. Then either $r \geq \operatorname{mr}(G)$ or $s \geq \operatorname{mr}(H)$.

Without loss of generality assume $r \geq \operatorname{mr}(G)$. By hypothesis $(\operatorname{mr}(G), 0) \in \mathcal{I}(G)$. Since $r+s-\operatorname{mr}(G)=\operatorname{mr}(H)$ then $(r-\operatorname{mr}(G), s) \in \mathcal{I}(H)$ as a point on the minimum rank line.

Let $A \in S(G)$ such that $\operatorname{pin}(A)=(\operatorname{mr}(G), 0)$ and let $B \in S(H)$ such that $\operatorname{pin}(B)=(r-\operatorname{mr}(G), s)$.

Let $n=|G \cup H|$. Define symmetric $n \times n$ matrices $\hat{A}=\left[\hat{a}_{i, j}\right]$ and $\hat{B}=\left[\hat{b}_{i, j}\right]$ by

$$
\begin{aligned}
& \hat{a}_{i, j}=\left\{\begin{array}{ll}
a_{i, j} & \text { if } i, j \in V(G) \\
0 & \text { otherwise }
\end{array},\right. \\
& \hat{b}_{i, j}=\left\{\begin{array}{ll}
b_{i, j} & \text { if } i, j \in V(H) \\
0 & \text { otherwise }
\end{array} .\right.
\end{aligned}
$$

Then $a \hat{A}+b \hat{B} \in S(G \cup H)$ for some $a, b \in \mathbb{R}^{+}$. Now

$$
\pi(a \hat{A}+b \hat{B}) \leq \pi(a \hat{A})+\pi(b \hat{B})=\pi(\hat{A})+\pi(\hat{B})=\pi(A)+\pi(B)=r .
$$

Similarly, $\nu(a \hat{A}+b \hat{B}) \leq s$. But,

$$
\begin{aligned}
r+s & =\operatorname{mr}(G)+\operatorname{mr}(H)=\operatorname{mr}(G \cup H) \leq \operatorname{rank}(a \hat{A}+b \hat{B}) \\
& =\pi(a \hat{A}+b \hat{B})+\nu(a \hat{A}+b \hat{B}) \leq r+s .
\end{aligned}
$$


Therefore, $r=\pi(a \hat{A}+b \hat{B})$ and $s=\nu(a \hat{A}+b \hat{B})$ which implies that $(r, s) \in$ $\mathcal{I}(G \cup H)$. Since $\mathcal{I}(G \cup H)$ contains the line $r+s=\operatorname{mr}(G)+\operatorname{mr}(H)$ by the Northeast Lemma, it contains $\mathrm{T}[\operatorname{mr}(G)+\operatorname{mr}(H),|G \cup H|]$.

EXample 8.2. Consider G158.

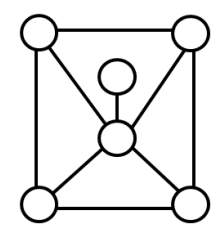

Since $G 158=W_{5} \cup K_{2}, \mathcal{I}\left(W_{5}\right)=\mathrm{T}[2,5], \mathcal{I}\left(K_{2}\right)=\mathrm{T}[1,2]$, and $\operatorname{mr}\left(W_{5} \cup K_{2}\right)=$ $\operatorname{mr}\left(W_{5}\right)+\operatorname{mr}\left(K_{2}\right)$, then by Theorem 8.1, $\mathcal{I}(G 158)=\mathrm{T}[3,6]$.

This theorem may be applied to determine the inertia sets of graphs G143, G158, G159, G172, G182, G184, G196, and G198. We list the decompositions for each below:

$G 143=G 48 \cup K_{2}, G 158=W_{5} \cup K_{2}, G 159=W_{5} \cup K_{2}, G 172=G 48 \cup K_{3}, G 182=$ $W_{5} \cup K_{3}, G 184=W_{5} \cup K_{3}, G 196=W_{5} \cup K_{4}$, and $G 198=G 190 \cup K_{2}$.

9. Inertia sets for all connected graphs on 6 or fewer vertices. We have now found the inertia sets for all connected graphs on 6 or fewer vertices but one. This graph is G174, also known as the 3-prism.

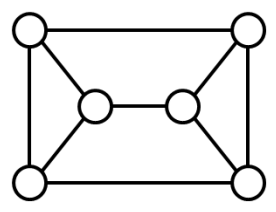

We note that $\operatorname{mr}(3$-prism $)=3$.

To find the inertia set of the 3 -prism we construct the following $6 \times 6$ matrix $B$, creating a matrix $A$ such that each off-diagonal entry of $A$ is nonzero.

$$
B=\left[\begin{array}{cc}
A & I \\
I & A^{-1}
\end{array}\right]
$$

Since $\left[\begin{array}{ll}I & A^{-1}\end{array}\right]=A^{-1}\left[\begin{array}{ll}A & I\end{array}\right]$ we have $\operatorname{rank}(B)=3$. We use two different matrices, $A$, and Observation 3.8 to obtain the possible points on the minimum rank line for $\mathcal{I}(3$-prism). 
The following matrix yields the axis points on the minimum rank line.

$$
A=\left[\begin{array}{lll}
2 & 1 & 1 \\
1 & 2 & 1 \\
1 & 1 & 2
\end{array}\right], \quad A^{-1}=\frac{1}{4}\left[\begin{array}{ccc}
3 & -1 & -1 \\
-1 & 3 & -1 \\
-1 & -1 & 3
\end{array}\right]
$$

Because $A$ is a positive definite matrix we know that $A$ has all positive eigenvalues. Then $\operatorname{pin}(A)=(3,0)$ and $\operatorname{pin}(-A)=(0,3)$.

To obtain the points $(2,1)$ and $(1,2)$ we use the following matrix $A$.

$$
A=\left[\begin{array}{lll}
0 & 1 & 1 \\
1 & 0 & 1 \\
1 & 1 & 0
\end{array}\right], \quad A^{-1}=\frac{1}{2}\left[\begin{array}{ccc}
-1 & 1 & 1 \\
1 & -1 & 1 \\
1 & 1 & -1
\end{array}\right]
$$

Because $\operatorname{det} A=2$ and trace $A=0, \operatorname{pin}(A)=(1,2)$. Then by Observation 3.8 we also obtain the point $(2,1)$.

Consequently, for the principal submatrix $A$ of $B, \operatorname{pin}(A)$ can take on the values $(3,0),(2,1),(1,2),(0,3)$. Since $\operatorname{rank}(B)=3$, it follows that the partial inertia of $B$ can be any of $(3,0),(2,1),(1,2),(0,3)$. Then by the Northeast Lemma, $\mathcal{I}(3$-prism) $=$ $\mathrm{T}[3,6]$.

The following table displays the inertia sets of all connected graphs with 6 or fewer vertices. Note that $n$ denotes the number of vertices in the graph. 
Table 9.1

\begin{tabular}{|l|l|}
\hline \multicolumn{1}{|c|}{$\mathcal{I}(G)$} & \multicolumn{1}{|c|}{ Graph } \\
\hline \hline $\mathrm{T}[0,1]$ & $\left.\mathrm{G} 1 K_{1}\right)$ \\
\hline $\mathrm{T}[1, n]$ & $\mathrm{G} 3, \mathrm{G} 7, \mathrm{G} 18, \mathrm{G} 52, \mathrm{G} 208,\left(K_{n}, n>1\right)$ \\
\hline $\mathrm{T}[n-1, n]$ & $\mathrm{G} 6, \mathrm{G} 14, \mathrm{G} 31, \mathrm{G} 83,\left(P_{n}, n>2\right)$ \\
\hline $\mathrm{T}[n-2, n]$ & $\mathrm{G} 16, \mathrm{G} 38, \mathrm{G} 105,\left(C_{n}, n>3\right)$ \\
\hline $\mathrm{T}[2,4]$ & $\mathrm{G} 15, \mathrm{G} 17$ \\
\hline $\mathrm{T}[2,5]$ & $\mathrm{G} 42, \mathrm{G} 45, \mathrm{G} 48-\mathrm{G} 51$ \\
\hline $\mathrm{T}[3,5]$ & $\mathrm{G} 34-\mathrm{G} 37, \mathrm{G} 40, \mathrm{G} 41, \mathrm{G} 43, \mathrm{G} 47$ \\
\hline $\mathrm{T}[2,6]$ & $\mathrm{G} 165, \mathrm{G} 190, \mathrm{G} 191, \mathrm{G} 194, \mathrm{G} 195, \mathrm{G} 199, \mathrm{G} 200, \mathrm{G} 203-$ \\
& $\mathrm{G} 207$ \\
\hline $\mathrm{T}[3,6]$ & $\mathrm{G} 117, \mathrm{G} 119, \mathrm{G} 126, \mathrm{G} 130, \mathrm{G} 133, \mathrm{G} 134, \mathrm{G} 140-\mathrm{G} 144$, \\
& $\mathrm{G} 150, \mathrm{G} 151, \mathrm{G} 153, \mathrm{G} 154, \mathrm{G} 156-\mathrm{G} 160, \mathrm{G} 163, \mathrm{G} 166$, \\
& $\mathrm{G} 168-\mathrm{G} 174, \mathrm{G} 177-\mathrm{G} 188, \mathrm{G} 192, \mathrm{G} 193, \mathrm{G} 196, \mathrm{G} 198$, \\
\hline $\mathrm{T}[4,6]$ & $\mathrm{G} 202$ \\
\hline $\mathrm{T} 93-\mathrm{G} 99, \mathrm{G} 102-\mathrm{G} 104, \mathrm{G} 111-113, \mathrm{G} 115, \mathrm{G} 118, \mathrm{G} 120$, \\
$\mathrm{T}[3, n] \cup\{(1,1)\}$ & $\mathrm{G} 122-124, \mathrm{G} 127, \mathrm{G} 128, \mathrm{G} 136, \mathrm{G} 137, \mathrm{G} 139, \mathrm{G} 147, \mathrm{G} 148$, \\
\hline $\mathrm{T}[4, n] \cup\{(2,1),(1,2)\}$ & $\mathrm{G} 13, \mathrm{G} 164, \mathrm{G} 4676, \mathrm{G} 175, \mathrm{G} 189, \mathrm{G} 197, \mathrm{G} 201$ \\
\hline $\mathrm{T}[4, n] \cup\left[\{(1,1)\}^{\prime}\right]_{n}$ & $\mathrm{G} 30, \mathrm{G} 92, \mathrm{G} 100, \mathrm{G} 114, \mathrm{G} 121, \mathrm{G} 125, \mathrm{G} 129, \mathrm{G} 135, \mathrm{G} 138$, \\
\hline $\mathrm{T}[5,6] \cup\left[\{(1,1)\}^{\prime}\right]_{6}$ & $\mathrm{G} 77, \mathrm{G} 146, \mathrm{G} 161$ \\
\hline $\mathrm{T}[5,6] \cup\left[\{(2,1),(1,2)\}^{\prime}\right]_{6}$ & $\mathrm{G} 78$ \\
\hline $\mathrm{T}[5,6] \cup\{(3,1),(2,2),(1,3)\}$ & $\mathrm{G} 79-\mathrm{G} 81$ \\
\hline &
\end{tabular}

10. Balanced inertia. The concept of balanced inertia was introduced by Barioli and Fallat [2].

Definition 10.1. A graph $G$ is said to have balanced inertia if there is a point $(r, s) \in \mathcal{I}(G)$ such that $|r-s| \leq 1$ and $r+s=\operatorname{mr}(G)$.

OBservation 10.2. If $\mathcal{I}(G)$ is a trapezoid, then $G$ has balanced inertia.

By examining Table 9.1 we have the following theorem.

TheOREM 10.3. All graphs on 6 or fewer vertices have balanced inertia.

Not all graphs are inertia balanced. See Theorem 7.3 in [4].

Proposition 10.4. If $G$ is a complete graph, a complete bipartite graph, or a clique-star, then $G$ has balanced inertia.

Proof. First, $(0,0)$ is in $\mathcal{I}\left(K_{1}\right)$. By Proposition 4.1, all complete graphs on 2 or 
more vertices have $(1,0) \in \mathcal{I}\left(K_{n}\right)$ and $\operatorname{mr}\left(K_{n}\right)=1$. By Theorem 4.2, all complete bipartite graphs have $(1,1) \in \mathcal{I}\left(K_{m, n}\right)$ and $\operatorname{mr}\left(K_{m, n}\right)=2$. By Lemma 4.3, all cliquestars have $(1,1) \in \mathcal{I}\left(K S_{m, n}\right)$ and $\operatorname{mr}\left(K S_{m, n}\right)=2$. Thus complete graphs, complete bipartite graphs, and clique-stars have balanced inertia.

TheOREM 10.5. Let $G$ be a graph on $n$ vertices. If $\operatorname{mr}(G)=\operatorname{cc}(G)$, then $G$ has balanced inertia.

Proof. By Theorem 4.6, $\mathcal{I}(G)=\mathrm{T}[\operatorname{mr}(G), n]$. By Observation 10.2, $G$ has balanced inertia.

TheOREM 10.6. Let $G$ be a graph with edge e and suppose $\operatorname{mr}\left(G_{e}\right)=\operatorname{mr}(G)+1$. If $G$ has balanced inertia, then so does $G_{e}$.

Proof. Let $(r, s) \in \mathcal{I}(G)$ with $r+s=\operatorname{mr}(G)$ and $|r-s| \leq 1$. By Theorem 6.1, $(r+1, s)$ and $(r, s+1)$ are contained in $\mathcal{I}\left(G_{e}\right)$. Further, $r+s+1=\operatorname{mr}\left(G_{e}\right)$. If $r \geq s$ then $|r-(s+1)| \leq 1$. If $r<s$ then $|r+1-s|=0$. Therefore, $G_{e}$ has balanced inertia.

TheOREM 10.7. Let $G$ be a graph with edge e and suppose $\operatorname{mr}(G \backslash e)=\operatorname{mr}(G)+1$. If $G$ has balanced inertia, then so does $G \backslash e$.

Proof. Let $(r, s) \in \mathcal{I}(G)$ with $r+s=\operatorname{mr}(G)$ and $|r-s| \leq 1$. By Theorem 6.6, $(r+1, s)$ and $(r, s+1)$ are contained in $\mathcal{I}(G \backslash e)$. Further, $r+s+1=\operatorname{mr}(G \backslash e)$. If $r \geq s$ then $|r-s+1| \leq 1$. If $r<s$ then $|r+1-s|=0$. Therefore, $G \backslash e$ has balanced inertia.

Theorem 10.8. If $G$ has balanced inertia, then so does $G \vee K_{1}$.

Proof. Let $(r, s)$ be in $\mathcal{I}(G)$ with $|r-s| \leq 1$ and $r+s=\operatorname{mr}(G)$. By Corollary 7.2, $\operatorname{mr}(G)=\operatorname{mr}\left(G \vee K_{1}\right)$ and by Theorem 7.1, $\mathcal{I}(G)=\left[\mathcal{I}\left(G \vee K_{1}\right)\right]_{n}$. Since $(r, s) \in$ $\mathcal{I}\left(G \vee K_{1}\right), G \vee K_{1}$ has balanced inertia.

TheOREm 10.9. If $\mathcal{I}(G)=\mathrm{T}[\operatorname{mr}(G),|G|], \mathcal{I}(H)=\mathrm{T}[\operatorname{mr}(H),|H|]$, and $\operatorname{mr}(G \cup H)=\operatorname{mr}(G)+\operatorname{mr}(H)$, then $G \cup H$ has balanced inertia.

Proof. By Theorem 8.1, $\mathcal{I}(G \cup H)=\mathrm{T}[\operatorname{mr}(G)+\operatorname{mr}(H),|G \cup H|]$. By Observation 10.2, $G \cup H$ has balanced inertia. $\square$

11. The Hermitian case. We now explain how to extend our results to the Hermitian case. We have elected to postpone this discussion to the penultimate section in order to avoid the awkwardness of including qualifying statements for the real symmetric and complex Hermitian case in the statements of every theorem. We recall the following definitions from [4] and [5]. 
Definition 11.1. Given a graph $G$ on $n$ vertices, let $\mathcal{H}(G)$ be the set of all complex Hermitian $n \times n$ matrices $A=\left[a_{i j}\right]$ such that $a_{i j} \neq 0, i \neq j$, if and only if $i j$ is an edge of $G$. Let $\mathcal{H}_{+}(G)$ be the subset of $\mathcal{H}(G)$ consisting of all positive semidefinite matrices in $\mathcal{H}(G)$. Then the Hermitian minimum rank of $G$ is

$$
\operatorname{hmr}(G)=\min _{A \in \mathcal{H}(G)}\{\operatorname{rank}(A)\} .
$$

and the positive semidefinite Hermitian minimum rank is

$$
\operatorname{hmr}_{+}(G)=\min _{A \in \mathcal{H}_{+}(G)}\{\operatorname{rank}(A)\} .
$$

The Hermitian inertia set of $G$ is

$$
\mathrm{h} \mathcal{I}(G)=\{(r, s) \mid \operatorname{pin}(A)=(r, s) \text { for some } A \in \mathcal{H}(G)\} .
$$

We will let $n$ be the number of vertices of $G$ throughout this section. We also recall Observations 2.1 and 2.2 from [4].

ObSERVATion 11.2. For any graph $G, \mathrm{~h} \mathcal{I}(G) \subseteq \mathrm{T}[\mathrm{hmr}(G), n]$.

ObSERvation 11.3. For any graph $G, \mathcal{I}(G) \subseteq \mathrm{h} \mathcal{I}(G)$ and $\operatorname{hmr}(G) \leq \operatorname{mr}(G)$. It is known that $\mathcal{I}(G)$ can be a strict subset of $\mathrm{h} \mathcal{I}(G)$ (see page 1184 of [4]).

We must now briefly refer to some results on zero forcing from [1]. (The definition of the zero forcing number will not be needed.) Proposition 2.4 and Proposition 4.3 are

Proposition 11.4. For any graph $G, M(G) \leq Z(G)$.

Proposition 11.5. For any graph $G$ with $|G| \leq 6, M(G)=Z(G)$.

Reformulating these two statements in terms of minimum rank we have

$$
\begin{gathered}
\operatorname{mr}(G) \geq n-Z(G) \text { for any graph } G, \\
\operatorname{mr}(G)=n-Z(G) \text { for any graph } G \text { with }|G| \leq 6 .
\end{gathered}
$$

From the discussion in Section 5 of [1], we also have

$$
\operatorname{hmr}(G) \geq n-Z(G) \text { for any graph } G \text {. }
$$

Consequently, if $G$ is a graph with $|G| \leq 6$,

$$
n-Z(G)=\operatorname{mr}(G) \geq \operatorname{hmr}(G) \geq n-Z(G) .
$$


So $\operatorname{mr}(G)=\operatorname{hmr}(G)$ for all graphs $G$ with $|G| \leq 6$. It follows that if $\mathcal{I}(G)$ is a trapezoid, we have

$$
\mathcal{I}(G)=\mathrm{T}[\operatorname{mr}(G), n]=\mathrm{T}[\mathrm{hmr}(G), n] \supseteq \mathrm{h} \mathcal{I}(G) \supseteq \mathcal{I}(G)
$$

and $\mathcal{I}(G)=\mathrm{h} \mathcal{I}(G)$.

We next consider graphs in Table 9.1 whose inertia sets are not trapezoids. First note that we can read off $\mathrm{mr}_{+}(G)$ from Table 9.1 from its "trapezoidal part" $\mathrm{T}[k, n]$. We have $\mathrm{mr}_{+}(G)=k$. By comparing these values against the values of $\mathrm{hmr}_{+}(G)$ in Table 4.1 of [6], it is straightforward to check that $\mathrm{mr}_{+}(G)=\mathrm{hmr}_{+}(G)$ for all graphs $G$ on 6 or fewer vertices. This is sufficient to guarantee that $h \mathcal{I}(G)=\mathcal{I}(G)$ for all graphs in Table 9.1 except possibly the last line. But graphs G79, G80, and G81 are trees. As noted on page 2 of $[4], \mathcal{I}(G)=\mathrm{h} \mathcal{I}(G)$ whenever $T$ is a tree. Thus we establish the following result.

TheOrem 11.6. If $G$ is a graph on 6 or fewer vertices, then $\mathcal{I}(G)=\mathrm{h} \mathcal{I}(G)$.

The Northeast Lemma and Observation 3.6 hold for $\mathrm{h} \mathcal{I}(G)$ as well as $\mathcal{I}(G)$ while Theorems 3.1 and 3.2 are true if we replace $\mathrm{mr}_{+}(G)$ by $\mathrm{hmr}_{+}(G)$. It follows that $\mathcal{I}(G)=\mathrm{h} \mathcal{I}(G)$ for complete graphs, complete bipartite graphs, and clique-stars.

A Hermitian form of Theorem 4.6 is obtained by replacing $\operatorname{mr}(G)$ by $\operatorname{hmr}(G)$ and $\mathcal{I}(G)$ by $\mathrm{h} \mathcal{I}(G)$. However, we do not know any graph for which $\operatorname{hmr}(G)<$ $\operatorname{mr}(G)=\operatorname{cc}(G)$. Theorem 4.8 is trivially true with $\mathcal{I}(G)$ replaced by $\mathrm{h} \mathcal{I}(G)$ because $\mathcal{I}(G) \subseteq \mathrm{h} \mathcal{I}(G)$.

If $G \neq K_{n}$ and $G^{c}$ is a disjoint union of complete bipartite graphs, we have $1<\operatorname{hmr}(G) \leq \operatorname{mr}(G)=2$ so that by Theorem 5.1,

$$
\mathrm{h} \mathcal{I}(G) \subseteq \mathrm{T}[\mathrm{hmr}(G), n]=\mathrm{T}[2, n]=\mathcal{I}(G) \subseteq \mathrm{h} \mathcal{I}(G) .
$$

It follows that Theorem 5.1 holds with $\mathcal{I}(G)$ replaced by $\mathrm{h} \mathcal{I}(G)$.

By examining the proof of Theorem 6.1, we see that if $A \in \mathcal{H}(G), A_{e} \in \mathcal{H}\left(G_{e}\right)$. It follows that Theorem 6.1 and its corollaries hold with each $\mathcal{I}$ replaced by $\mathrm{h} \mathcal{I}$. By similar reasoning, the same holds for Theorem 6.6 and its corollaries.

Theorem 7.1 and its corollary remain true if each $\mathcal{I}$ is replaced by $\mathrm{h} \mathcal{I}$ and $\mathrm{mr}$ and $\mathrm{mr}_{+}$are replaced by $\mathrm{hmr}$ and $\mathrm{hmr}_{+}$, respectively. In the proof of the reverse containment of Theorem 7.1, the argument takes place in $\mathbb{C}^{n}$ instead of $\mathbb{R}^{n}$.

Theorem 8.1 is also valid for $\mathrm{h} \mathcal{I}(G)$ provided each $\mathcal{I}$ is replaced by $\mathrm{h} \mathcal{I}$ and each $\mathrm{mr}$ is replaced by $\mathrm{hmr}$.

The definition of balanced Hermitian inertia is the same as that for balanced inertia with $\mathcal{I}(G)$ replaced by $h \mathcal{I}(G)$. Of course, Observation 10.2 remains true for 
$\mathrm{h} \mathcal{I}(G)$. Because of Theorems 11.6 and 10.3, all graphs on 6 or fewer vertices have balanced Hermitian inertia. The Hermitian analogues of Proposition 10.4 and the remaining five theorems in Section 10 are valid as well.

We conclude that within the scope of this paper there is no material difference between $\mathcal{I}(G)$ in the real symmetric case and $\mathrm{h} \mathcal{I}(G)$ in the complex Hermitian case. One simply has to make the obvious notational changes and all preceding theorems remain valid.

12. Conclusion. We have successfully determined the inertia sets for all graphs on six or fewer vertices using the techniques mentioned in the paper. However, we note that there are multiple ways to calculate some of the inertias, both with our own and with previously known methods. For example, almost half of the connected graphs on 6 or fewer vertices have a cut vertex so we could have used the reduction formula, Theorem 4.2 in [4], to calculate the inertia set of each of these in terms of the inertia sets of smaller graphs. However, this method is somewhat cumbersome to use by hand. We could have also used Theorem 6.1 in [4] to determine the inertia sets of all trees, but there were so few that this was not needed. By introducing several new techniques, we were able to determine the inertia sets for all connected graphs on six and fewer vertices by more efficient methods. These new techniques can be applied to a large number of graphs with more than six vertices. However, despite the extensive possibilities of these techniques, they do have their limitations.

The Edge Subdivision Theorem for Partial Inertias has an important limitation. We know what happens to the inertia set if we subdivide an edge and the minimum rank increases. However, when the minimum rank remains the same, the theorem no longer applies and we must rely on other methods. Graphs G121, G125, G149 are examples of graphs whose minimum rank did not change when an edge was subdivided. Fortunately, these graphs are sufficiently small that we could compute their inertia sets in spite of being unable to use the Edge Subdivision Theorem. For larger graphs the problems may become more frequent and difficult to handle. For example, consider the graph $K_{4}$ and subdivide every edge of it once. The minimum rank of this fully subdivided graph is the same as the minimum rank of the graph with exactly five edges subdivided. It follows that the inertia set for the fully subdivided graph cannot be determined by any of our existing techniques and new methods must be developed. We summarize this as the following question.

Question 12.1. If $\operatorname{mr}\left(G_{e}\right)=\operatorname{mr}(G)$, how is $\mathcal{I}\left(G_{e}\right)$ related to $\mathcal{I}(G)$ ?

A related question is

QUESTION 12.2. If $\operatorname{mr}\left(G_{e}\right)=\operatorname{mr}(G)$ and $G$ has balanced inertia, does $G_{e}$ have balanced inertia? 
The edge deletion technique works well when the minimum rank of a graph increases but it also has its limitations. This method fails to be useful when the minimum rank goes down or remains the same after an edge has been deleted. In this instance, little can be said about the inertia of the new graph. An analogue to Question 12.2 for edge deletion is

QUESTION 12.3. If $\operatorname{mr}(G \backslash e)=\operatorname{mr}(G)$ and $G$ has balanced inertia, does $G \backslash e$ have balanced inertia?

The clique cover technique works well in the case that the minimum rank equals the clique cover number, but is no longer effective when the minimum rank is less than the clique cover number. For example, consider $W_{6}$. The clique cover number is 5 but the minimum rank is 3 . Using only the clique cover number to determine the inertia set, we find that we miss both the 4 -line and the 3 -line. When the clique cover number is an overestimate of the minimum rank of a graph, we must again rely on other methods to compute its inertia set. On six vertices, this task was not difficult because the graphs were frequently trees and it is known how to compute the inertia sets for these. For graphs of larger order, it is difficult to calculate the inertia sets using only the clique cover number.

The clique/clique-star cover was a useful asset to our set of techniques. In instances that the clique cover number broke down, we found a combination of cliques and clique-stars that yielded the minimum rank as well as the entire inertia set by applying Theorem 4.8. However, we do not know how effective this cover will be for larger graphs. For example, consider the following graph:

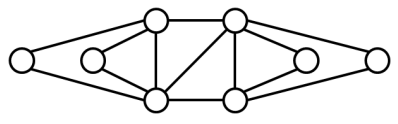

There are several ways we can cover this graph. We can use 2 clique-stars, a cliquestar and 3 cliques, or 6 cliques. By applying Theorem 4.8, we can determine points in the graph's inertia set. Doing so gives us that $\mathrm{T}[6,8] \cup\left[\{(2,2),(4,1),(1,4)\}^{\nearrow}\right]_{8}$ is contained in the inertia set. However, we do not know whether the theorem gives us all the points in that set. The points $(3,1)$ and $(1,3)$ are not given by the theorem but we do not have a method to say that those points cannot be in the inertia set. This example motivates our penultimate question:

QUESTION 12.4. For what class of graphs can we determine the entire inertia set by Theorem 4.8?

In addition to exploring the limitations of our methods, we have developed further questions by examining Table 9.1 . We notice that in the table every graph for which $\mathcal{I}(G)$ is not a trapezoid contains $K S_{1,3}\left(K_{1,3}\right)$ as an induced subgraph. In graph theory literature, this graph is frequently referred to as a claw, and the properties of 
claw-free graphs have been intensely investigated. Our final question is

QUESTION 12.5. If $G$ is a claw-free graph, is $\mathcal{I}(G)$ a trapezoid?

\section{REFERENCES}

[1] AIM Minimum Rank-Special Graphs Work Group. Zero forcing sets and the minimum rank of graphs. Linear Algebra and its Applications, 428:1628-1648, 2008.

[2] F. Barioli and S. Fallat. On the minimum rank of the join of graphs and decomposable graphs. Linear Algebra and its Applications, 421:252-263, 2007.

[3] W. Barrett, R. Bowcutt, M. Cutler, S. Gibelyou, K. Owens. Minimum rank of edge subdivisions of graphs. Electronic Journal of Linear Algebra, 18:530-563, 2009.

[4] W. Barrett, H. Tracy Hall and R. Loewy. The inverse inertia problem for graphs: Cut vertices, trees, and a counterexample. Linear Algebra and Its Applications, 431:1147-1191, 2009.

[5] W. Barrett, H. van der Holst and R. Loewy. Graphs whose minimal rank is two. Electronic Journal of Linear Algebra, 11:258-280, 2004.

[6] M. Booth, P. Hackney, B. Harris, C. R. Johnson, M. Lay, L. H. Mitchell, S. K. Narayan, A. Pascoe, K. Steinmetz, B. D. Sutton, and W. Wang. On the minimum rank among positive semidefinite matrices with a given graph. SIAM Journal on Matrix Analysis and Applications, 30:2:731-740, 2008.

[7] Hein van der Holst. On the maximum positive semi-definite nullity and the cycles matroid of graphs. Electronic Journal of Linear Algebra, 18:192-201, 2009.

[8] C. R. Johnson, R. Loewy, and P. A. Smith. The graphs for which the maximum multiplicity of an eigenvalue is two. Linear and Multilinear Algebra, 57:713-736, 2009.

[9] P. M. Nylen. Minimum-rank matrices with prescribed graph. Linear Algebra and its Applications, 248:303-316, 1996.

[10] R. C. Read and R. J. Wilson. An Atlas of Graphs. Oxford University Press, New York, 1998. 